\title{
Radarbilder für die Geowissenschaften
}

\section{Einleitung}

Die Raumfahrt hat mittlerweile den Ruf des Spektakulären verloren. Ihre Anwendung, insbesondere bei der Übermittlung von Nachrichten über interkontinentale Distanzen, aber auch zur Gewinnung von Informationen über die Atmosphäre und die Erdoberfläche, sind zu einer Selbstverständlichkeit in unserem täglichen Leben geworden. Satellitenbilder sind heute jedermann geläufig als Grundlage der Wettervorhersage im abendlichen Fernsehprogramm, als didaktisches Gestaltungsmittel im Geographieunterricht oder als Aufnahmen des amerikanischen LANDSAT-Satelliten, die großflächige Landnutzungskartierungen sowie Erdöl- und Mineralprospektionen ermöglichen. Die meisten Bilder der Erdoberfläche aus der Luft sind bis heute entweder auf die uns bekannte Art der Photographie oder Multispektralscanner-Technik aufgenommen worden, d.h. durch das Fokussieren von reflektiertem sichtbarem Sonnenlicht auf eine lichtempfindliche Schicht eines Filmes, resp. auf lichtempfindliche Detektoren. Andere Fernerkundungstechniken basieren auf der Erfassung von thermischer Strahlung oder Mikrowellenstrahlung, die von der Erdoberfläche abgestrahlt und mit Hilfe von Scannern und Radiometern registriert wird.

Obwohl wir in den nächsten Jahren mit qualitativ immer besseren Bildern rechnen dürfen, haftet der optischen Fernerkundung im sichtbaren und infraroten Wellenbereich (Fig. 1) ein großer Nachteil an: Bei ungünstigen Witterungsbedingungen ist der Erfolg dieser Sensoren z. T. oder auch ganz in Frage gestellt, da die von der Erde reflektierte und abgestrahlte Energie durch Dunst oder Wolken abgeschwächt oder ganz absorbiert wird. Dabei wäre die Möglichkeit der kontinuierlichen Beobachtung (d.h. Aufnahmen in bestimmten Zeitabständen) von entscheidender Bedeutung für das Erfassen von dynamischen Prozessen auf der Erdoberfläche wie z. B.:

- Intensität und Richtung von Meeresströmungen, Lokalisierung von Ölteppichen nach Ölunfällen, Optimierung der Schiffsrouten auf den Weltmeeren,

- Zustand der Vereisung von arktischen Gewässern und die Bewegung von Eisbergen für die Schiffsnavigation,

- Landnutzungsveränderungen in tropischen Gebieten, z.B. übermäßiger Kahlschlag in tropischen Wäldern.
Da in vielen Gebieten eine Überwachung mit konventionellen Mitteln wegen der ständigen Wolkendecke unmöglich ist, werden dauernd neue Methoden und Techniken gesucht.

Ein Ausweichen auf den Mikrowellenbereich des elektromagnetischen Spektrums bringt fürs erste auch nicht viel. Passive Systeme erreichen nämlich wegen den sehr kleinen Energiemengen, die von der Erde in diesem Wellenbereich abgestrahlt werden, nur eine schlechte räumliche Auflösung im Vergleich zu den hochauflösenden Aufnahmesystemen im sichtbaren und im daran anschließenden Infrarot-Bereich.

Um im Mikrowellenbereich Aufnahmen der Erde aus der Luft oder aus dem Weltraum von photoähnlicher Qualität zu erhalten, bedurfte es einer fundamental neuen Technik. Die Anforderungen an ein System, das jederzeit, also bei Tag und Nacht und ohne Rücksicht auf Wetterbedingungen, aus dem Weltall Bilder von der Erdoberfläche liefern kann, sind einfach: Um vom Sonnenlicht unabhängig zu sein, benötigt es seine eigene Beleuchtungsquelle und um die Wolken durchdringen zu können, eine Strahlung oder Wellenlänge, die vom Wasserdampf nicht oder aber nur wenig gedämpft wird. Das bilderzeugende System, das diese Anforderungen erfült, heißt RADAR und arbeitet meistens mit Wellenlängen zwischen $1 \mathrm{~cm}$ und $30 \mathrm{~cm}$, d. h. Frequenzen von $30 \mathrm{GHz}$ bzw. $1 \mathrm{GHz}$. Radar ist ein Akronym, abgeleitet vom englischen Radio Detection and Ranging. Radargeräte waren schon seit Beginn ihrer Entwicklung als Entfernungsmesser und Detektoren fliegender Objekte gedacht. Ich möchte mich nun aber nicht weiter dem sog. Ortungsradar widmen, der z. B. bei der Flugsicherung eines jeden Flughafens operationell benützt wird, sondern zeigen, wie man sich dieses Prinzipes bedient hat, um photoähnliche Bilder eines überflogenen Terrains zu erzeugen. Es geht hier nicht mehr um das relativ einfache Problem, die gut reflektierenden Metallteile eines Flugzeuges vor einem einheitlichen Hintergrund zu erfassen, sondern um die Abbildung der realen Wirklichkeit am Boden, bei der solch hohe Kontraste auf einem Bild nicht nur einmal, sondern millionenfach vorkommen.

Daniel Nüesch, PD Dr.,

Geographisches Institut Universität Zürich-Irchel, Winterthurerstr. 190, 8057 Zürich 


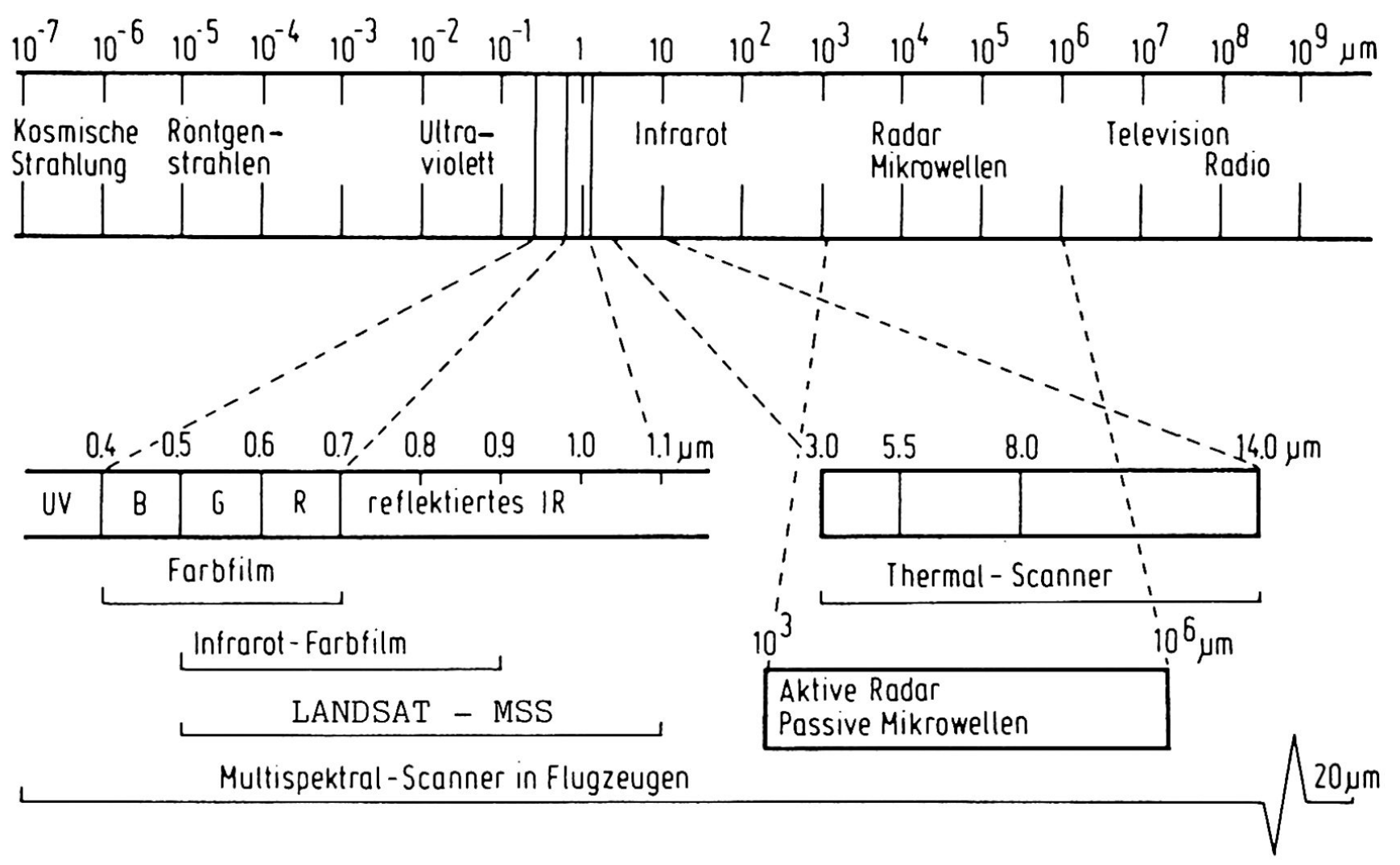

Fig.1 Elektromagnetisches Spektrum und Wirkungsbereich der gebräuchlichsten Fernerkundungssysteme

Diese neue Technik wurde ursprünglich für den Einsatz an Bord von Flugzeugen entwickelt. Die Radarantenne ist seitlich am Flugzeug angebracht. Über sie wird ein Hochfrequenzsignal abgestrahlt. Das Empfangssignal, das über die gleiche Antenne empfangen wird, besteht aus der von der Bodenoberfläche reflektierten Radarstrahlung. Nach entsprechender Verstärkung und Umsetzung über eine Kathodenstrahlröhre wird aus dem Empfangssignal ein Bildsignal erzeugt. Da es gilt, jedes Einzelziel am Boden zweidimensional zuzuordnen, ist es naheliegend, die eine Dimension mit der klassischen Radarfunktion der Entfernungsmessung abzudecken. Signale von näher gelegenen Zielen werden früher von der Antenne empfangen, als Signale von weiter entfernten Zielen. Die Auflösung quer zur Flugrichtung, d. h. die Tatsache, daß 2 Ziele am Boden vom System als 2 einzelne Ziele erkannt werden können, ist v. a. eine Funktion von möglichst kurzen Pulsen und vom Depressionswinkel, d. h. vom Winkel, unter welchem das Gelände beleuchtet wird. Je kürzer die Pulse sind und je weiter das Objekt vom Sensor entfernt ist, um so besser wird die Auflösung sein. Die Auflösung im Azimut, d.h. in Flugrichtung, ist eine Funktion der Antennenlänge und der Distanz zwischen dem Sensor und dem aufgenommenen Objekt. Ohne nun die genauen Formeln zu erläutern, für die ich auf Spezialliteratur verweisen möchte (ULABY et al., 1981; COLWELL, 1983), kann man hier festhalten, daß mit zunehmender Antennenlänge und abnehmender Entfernung zwischen Objekt und Sensor die geometrische Auflösung am Boden besser wird. Typische Auflösungswerte von einem in $10 \mathrm{~km}$ Höhe fliegenden Flugzeug betragen um die $20 \mathrm{~m}$ am Boden. Solche Systeme bezeichnet man als Radar mit realer Apertur (Fig. 2).

\section{REAL APERTURE RADAR}

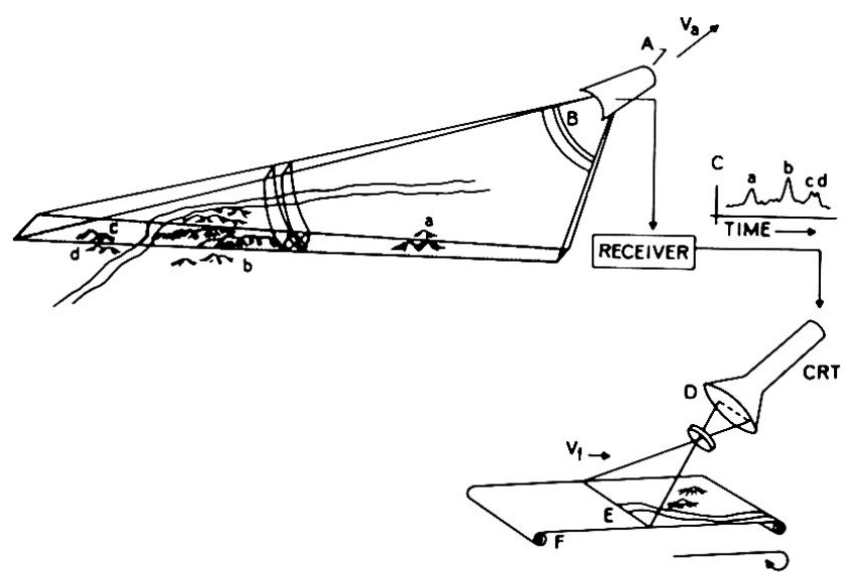

Fig. 2 Radar mit realer Apertur

Es leuchtet sicher jedermann ein, daß bei der Beobachtung vom Satelliten aus nicht mehr so gute Werte erreicht werden können. Wollte man mit dem Prinzip der realen Apertur gute Auflösungsleistungen erzie- 
len, müßte der Satellit mit einer über $2 \mathrm{~km}$ langen Antenne ausgerüstet sein, was schlicht unmöglich ist. Eine Lösung dieses Problems bietet die Anwendung des Prinzipes der synthetischen Apertur, kurz SAR genannt. SAR steht für Synthetic Aperture Radar. Darunter versteht man den Aufbau oder die Synthese einer großen Apertur mittels einer relativ kleinen Einzelantenne (Fig. 3). Dieses Prinzip verlangt, daß die Antenne längs einer bekannten, möglichst geradlinigen Bahn geführt wird, daß kurze kohärente Pulsfolgefrequenzen ausgestrahlt werden und $d a ß$ die empfangenen Radarsignale während des Fluges für die weitere Verarbeitung nach Betrag und Phase aufgezeichnet werden.

Genau wie im Falle der realen Apertur können aufgrund der unterschiedlichen Echo- oder Empfangszeiten die verschiedenen Punkte am Boden quer zur Flugrichtung entfernungsmäßig unterschieden werden. Um eine besonders hohe Auflösungsleistung quer zur Flugrichtung zu erreichen, mußte man sich etwas anderes einfallen lassen, als immer kürzere Pulse auszusenden, da diese z. B. im Nanosekundenbereich wegen der vorhandenen Systembandbreite zu wenig Leistung aufbrächten. Anstelle ganz kurzer Pulse werden längere ausgesendet, die ihre Frequenz pro Zeiteinheit über eine gegebene Systembandbreite linear ändern. Man nennt diese längeren Pulse auch Chirp-Signale. Bei der Verarbeitung der empfangenen Signale können die ursprünglichen Pulse mit Hilfe von "matched-Filtern" wieder komprimiert werden, um die gewünschte Auflösung zu erhalten. Auf diese Weise umgeht man die bei sehr kurzen Sendeimpulsen notwendige hohe Sendeleistung, die man an Bord eines Flugzeuges oder eines Satelliten nicht oder nur schwer erreichen kann. Um eine hohe Auflösung in Azimut- oder Flugrichtung zu erzielen, bedient man sich der Tatsache, daß durch die mehrfache Beleuchtung eines Zieles Doppler-Effekte auftreten, die sich in einer permanenten Frequenzänderung des Zieles äußern. Infolge der konstanten Geschwindigkeit des Flugzeuges kann jedem Ziel am Boden eine bestimmte Dopplerverschiebung zugeordnet werden. Echos von Zielen, die senkrecht zur Flugrichtung liegen, haben eine Dopplerverschiebung von Null. Ziele, die vor dieser Senkrechten liegen, verzeichnen eine Verschiebung zu höheren Frequenzen, diejenigen hinter der Senkrechten eine Verschiebung zu niedrigeren Frequenzen. Wenn diese zurückgestreuten Wellen zusammen kombiniert werden, interferieren sie miteinander entsprechend ihrer Amplitude und Phase. Das Interferenzmuster enthält dann die detaillierte Information des beleuchteten Streifens am Boden.

Jedes empfangene Echo (und während einer Mission sind dies Milliarden) wird zuerst in Zeitintervalle (engl. range gates) unterteilt und danach in einer Dopplerfilterbank in Azimutstreifen zerlegt. Am Ausgang der Dopplerfilterbank können sodann die Energieanteile der Echos gemessen werden, die von

\section{SYNTHETIC APERTURE RADAR}

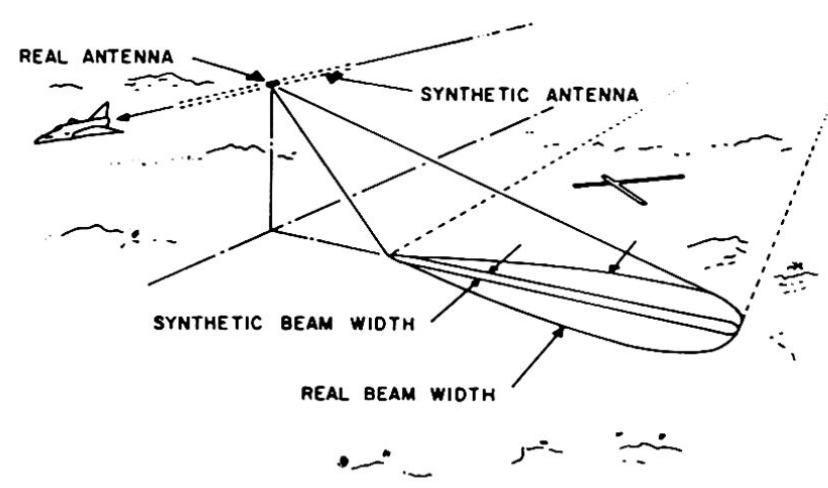

Fig. 3 Radar mit synthetischer Apertur

einem definierten Flächenstück (engl. Pixel = picture element) am Boden reflektiert wird. Damit die Amplitude und die Phase der Echosignale gemessen werden können, vergleicht man sie mit einem stabilen Referenzsignal. Eine ähnliche Technik kommt in der Radioastronomie zur Anwendung: Dabei wird eine lange Basis von Interferometern gebildet, indem man die Signale eines Arrays von weitauseinanderliegenden Radioteleskopen kombiniert.

\section{Verarbeitung von SAR-Daten}

Die Verarbeitung von SAR-Daten kann auf optischem oder digitalem Weg erfolgen. Bei der optischen Verarbeitung müssen die analogen zurückgestreuten Signale zuerst von einer Kathodenstrahlröhre in Lichtstrahlen entsprechender Helligkeitsschwankungen umgesetzt werden, die ihrerseits einen synchron mit der Frequenzbewegung entlang des abzubildenden Geländestreifens durchlaufenden Filmstreifen, den sog. "Datenfilm», belichten. Dieser Film entspricht nicht einem uns geläufigen Bild, denn es sind nur Amplituden und Phasen der reflektierten Signale nach Art eines Holograms gespeichert, bei dem jedes Ziel eine charakteristische Figur (Phasenfigur) von konzentrischen Ellipsen ergibt. Anschließend durchläuft dieser Datenfilm ein optisches Prozessorsystem, bestehend aus Laserquelle als Referenzstrahl und einer aufwendigen Linsen- und Filterkombination, die nach holographischem Prinzip den Signal-Historie genannten, über die azimutale Antennenkeulenbreite entstandenen Dopplersignalverlauf zum zugehörigen Bildlichtstrahl komprimiert. Dieses System, auch optischer Korrelator genannt, belichtet dann Punkt für Punkt einen ebenfalls synchron mitlaufenden sog. «Bildfilm», der nach anschließender Entwicklung zuerst das Negativbild und nach photographischem Umkopieren den abgebildeten Geländestreifen zeigt. Im Gegensatz zum optischen Verfahren kann das SAR-Bild auch auf digitalem, d.h. rechnerischem 
Weg erzeugt werden. Bei den Rechenoperationen handelt es sich um eine zweidimensionale Filterung (mathematisch eine Faltung) der Signalmatrix mit einer Filtermatrix als Referenzfunktion. Die Filtermatrix wird so gewählt, daß alle Beiträge eines Punktzieles zu einem Bildpunkt komprimiert werden. Man spricht auch von digitaler Korrelation im Gegensatz zur optischen Korrelation. Im Falle von SEASAT (dem ersten 1978 von der NASA für ozeanographische und meteorologische Studien in den Weltraum lancierten Satelliten) waren z. B. gegen 1000 komplexe Operationen notwendig, um die große Apertur zu synthetisieren und den Energie- oder Helligkeitsanteil eines Bildelementes, d.h. eines $25 \times 25 \mathrm{~m}$ großen Flächenstückes, am Boden zu bestimmen. Der Satellit bewegte sich in ca. $800 \mathrm{~km}$ Höhe mit einer Geschwindigkeit von $7,5 \mathrm{~km} / \mathrm{sec}$, und der Sensor bedeckte einen Streifen von $100 \mathrm{~km}$ Breite auf der Erdoberfläche, so daß ungefähr $10^{9}$ Operationen notwendig waren, um ein Bild jener Information zu erzeugen, die während einer einzigen Sekunde gesammelt wurde. Zusätzlich hatte das Verarbeitungssystem noch die durch die Erdrotation bedingten Dopplerverschiebungen und die durch die Erdkrümmung bedingten Verzerrungen sowie jene Verzerrungen, die durch kleine Variationen des Flugweges der Sensorplattform hervorgerufen wurden, zu berücksichtigen. - Die größte Herausforderung bei der Verarbeitung von Synthetic Aperture Radar-Daten liegt darin, herauszufinden, wie diese bereits im Weltraum korreliert werden könnten, um die ohnehin schon hoffnungslos überlasteten Datenübermittlungskanäle zu entlasten. Ziel wäre also die Übertragung eines fixfertigen Bildes und nicht der ungefilterten riesigen Signalmatrix. Die digitale Verarbeitung, die vorläufig noch nicht an Bord des Satelliten stattfindet, liefert zwar heute schon die qualitativ besseren Bilder, aber nur bei bedeutend höherem finanziellem Aufwand und langsamerer Produktionsrate als beim optischen Prozeß. Der CRAY X-MP, einer der heute (1984) schnellsten Rechner, ist in der Lage, ca. 420 Millionen Operationen pro Sekunde auszuführen. Dies ist aber immer noch über einen Faktor 2 weniger, als für die EchtzeitVerarbeitung der SEASAT-Daten erforderlich gewesen wäre. Der schnellste heute verfügbare Prozessor, der an Bord des Satelliten (also nicht in einem Rechenzentrum am Boden) die Radardaten während des Fluges hätte verarbeiten können, besitzt eine Durchschubrate von $1: 500$, d.h. daß die während einer Sekunde registrierten Daten noch 500 Sekunden Verarbeitungszeit erfordern. Dies hat zur Folge, daß die Rohdaten immer noch auf Bändern mit hoher Speicherdichte, sog. High Density Digital Recorder (HDDR), gespeichert werden müssen und, daß wegen der beschränkten Speicherkapazität an Bord des Satelliten pro Erdumlauf nur während maximal 5-10 Minuten Daten aufgenommen werden können. Ein Prozessor, der die Datenrate eines orbitalen SARSensors in Echtzeit verkraften kann, wird zur Zeit am
Jet Propulsion Laboratory in Pasadena, Kalifornien, gebaut und sollte auf Ende 1985 fertiggestellt sein. Die Anforderungen an einen sog. real time processor an Bord eines Satelliten sind ganz besonderer Art: kleines Volumen, minimaler Energieverbrauch und ohne Unterhalt über Jahre funktionstüchtig. Da die Rechenleistung eines solchen Prozessors, der übrigens speziell für die Radardatenverarbeitung entwikkelt und nicht als "general purpose computer» konzipiert sein müßte, auf das Mehrfache eines IBM3033-Systems veranschlagt wird, erstaunt es nicht, daß heute noch nicht mehrere solche Prozessoren im All in Betrieb stehen.

\section{Bildinterpretation}

Auf den ersten Blick sehen SAR-Bilder einem Bild aus einer konventionellen Kamera oder einem Multispektral-Scanner sehr ähnlich, v. a. bei Betrachtung auf größere Distanz (Abb.1). Bei genauerer Prüfung zeigen sich aber entscheidende Unterschiede: Das Relief ist in einer uns völlig ungewohnten Art wiedergegeben. Der Interpret muß hier berücksichtigen, daß das Radargerät ursprünglich nicht als abbildendes System konzipiert worden war und deshalb nur Laufzeiten der Radarpulse mißt, also Schräginformation liefert. Für viele Auswertungen, z. B. kartographische Anwendungen, ist deshalb eine sehr aufwendige Transformation in die gewünschte Projektionsebene notwendig. Diesen komplexen Problemen widmet sich heute ein neuer, eigens dafür geschaffener Wissenschaftszweig, die Radargrammetrie, die sich mit der Geometrie des Radarbildes befaßt.

Ein weiterer wesentlicher Unterschied zu den Aufnahmen im sichtbaren und infraroten Wellenbereich ist der, daß Radarbilder stark verrauscht sind. Betrachten wir das Bild aus einiger Entfernung, fällt uns nichts Besonderes, aus der Nähe aber sofort die Körnigkeit des Bildes auf. Genau dasselbe erleben wir beim Betrachten einer gerasterten Druckvorlage mit einer Lupe. - Fragen wir uns, woher diese Erscheinung rührt, müssen wir uns daran erinnern, wie das Signalecho zustande kommt, das registriert und gespeichert wurde. Da sich die modernen Radarsensoren kohärenter Strahlung bedienen, variiert das Rückstreusignal (auch wenn es sich nach menschlichem Ermessen um eine homogene Oberfläche handelt) je nach Phasenwinkel, indem es beim Empfänger ankommt. Man spricht von konstruktiver oder destruktiver Kohärenz. Beim Wert eines jeden Bildpunktes handelt es sich um ein statistisches Signal, das durch die komplizierte SAR-Verarbeitungsmethode ermittelt wurde. Dieses Rauschphänomen wird mit «speckle» bezeichnet. Im Englischen wird auch der Ausdruck "salt and peppen» (Salz und Pfeffer) verwendet. Im allgemeinen ist dieses Rauschen so stark, daß andere Rauscheffekte fast vernachlässigt werden können. 
Suchen wir konkret nach Möglichkeiten, diese statistisch variierende Rückstreuung in den Griff zu bekommen, dann halten wir heute noch vergeblich Ausschau nach Patentrezepten, wie sie z.T. für die automatische Klassifizierung von Fernerkundungsdaten im sichtbaren und infraroten Bereich bereits vorhanden sind. Der Grund dafür ist, wie wir schon vorhin angedeutet haben, darin zu suchen, daß flächenhafte Objekte, die wir mit unseren Augen als mehr oder weniger homogen betrachten, in Radarbildern noch weniger als z.B. in photographischen Aufnahmen durch einen konstanten und typischen Grauwert dargestellt sind. Wir können somit auf keinen Fall am absoluten Grauwert interessiert sein, sondern nur an der statistischen Erfassung der flächenhaften Verteilung der Grautonwerte mit ihren allfälligen Regelmäßigkeiten und gegenseitigen Abhängigkeiten innerhalb begrenzter Bildbereiche. Genau das ist das Forschungsziel der Texturanalyse. Sie läßt sich prinzipiell nicht punktbezogen in bezug auf die Bilddaten durchführen, sondern erfordert die zahlenmäßige Erfassung und Beschreibung der Grauwertstatistik in einzelnen Bildausschnitten. Aus Platzgründen kann ich in diesem Artikel nicht auf die spezielle Problematik der digitalen Texturanalyse eingehen. Sie ist u. a. in den angeführten Publikationen (HARALICK et al., 1973; CONNERS et al., 1980) behandelt worden.

Im Anschluß an diese theoretischen Ausführungen möchte ich anhand eines Beispieles aufzeigen, wie zur Zeit daran gearbeitet wird, diese neueste Technologie in Verbindung mit modernen Bildverarbeitungstechniken im meist bewölkten Raum der Arktis anzuwenden. Die kanadische Regierung sowie verschiedene private Firmen sind ganz besonders daran interessiert, die rohstoffreichen Gebiete der Nordwestpassage und in der Beaufort Sea im kommenden Jahrzehnt zu erschließen. Dafür sind vorerst noch umfangreiche Abklärungen erforderlich; so u.a. hinsichtlich der Überwachung von Eiszustand und Eisbewegung auf den Schiffahrtsrouten, aber auch für die Sicherheit der Bohrplattformen. Neben der Vorverarbeitung der Radardaten und der Klassierung von Flächen mit offenem Wasser, jungem oder älterem, mehrjährigem Eis gilt es, eine Methode zu finden, die ein Klassifikationsresultat in Echtzeit, d. h. also innert Sekunden bis Minuten, zuhanden der Schiffscrew liefern kann. Im ersten Bild (Abb. 2) sind die Bilddaten eines Radars zu sehen, der im X-Band arbeitete, die $3 \mathrm{~cm}$ langen Wellen horizontal aussendete und nur den vertikalen Polarisationsanteil des Rückstreusignals empfing. Die Daten stammen von der im März 1979 in der Beaufort Sea als Teil des kanadischen SURSAT-Projektes durchgeführten SAR-580-Kampagne. Man erkennt deutlich den hohen dynamischen Bereich der Empfangssignale: die weissen Stellen deuten auf Orte, an denen die Radarwellen intensiv zurückgestreut wurden. Das läßt auf eine stark strukturierte Oberfläche schließen, also auf mehrjähriges Eis. Die dunklen

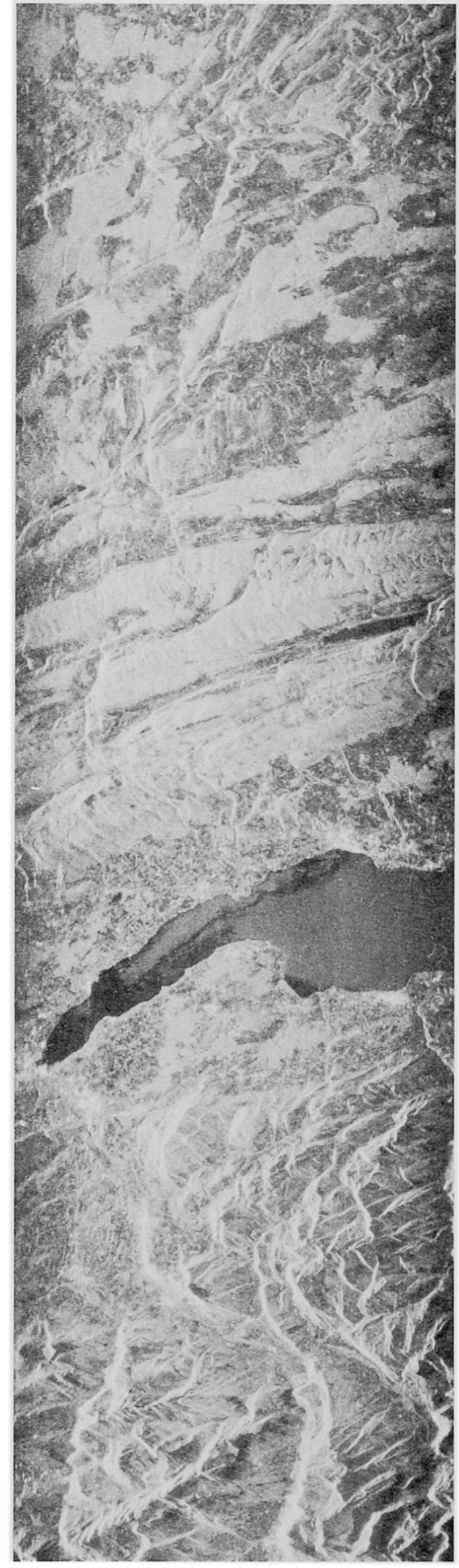

Abb. 1 SEASAT-SAR-Aufnahme vom 19. Aug. 1978 über dem Gebiet des westlichen Genfersees 


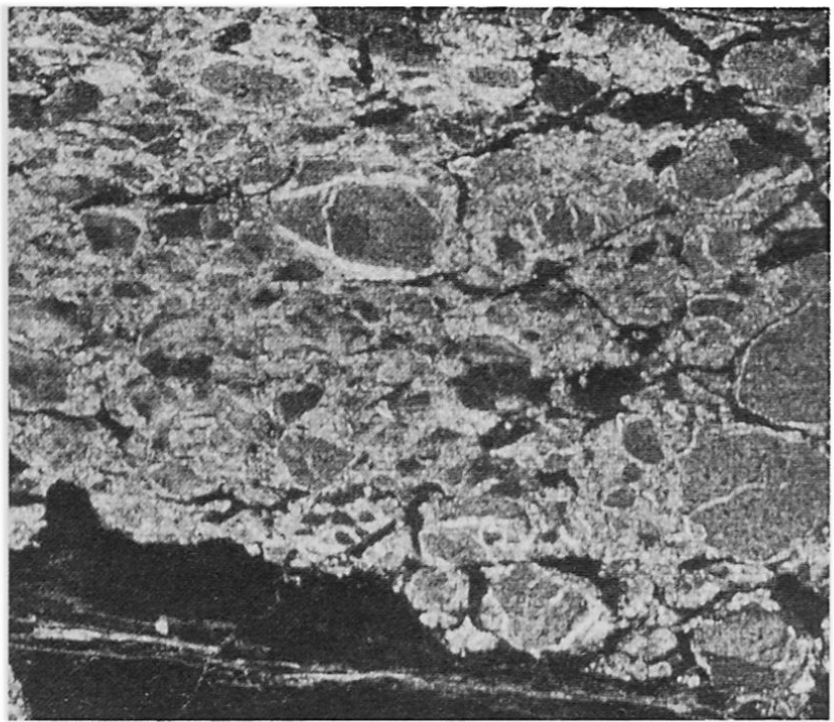

Abb. 2 Original-SAR-Daten von Meereis in der Beaufort Sea (X-Band, HV)

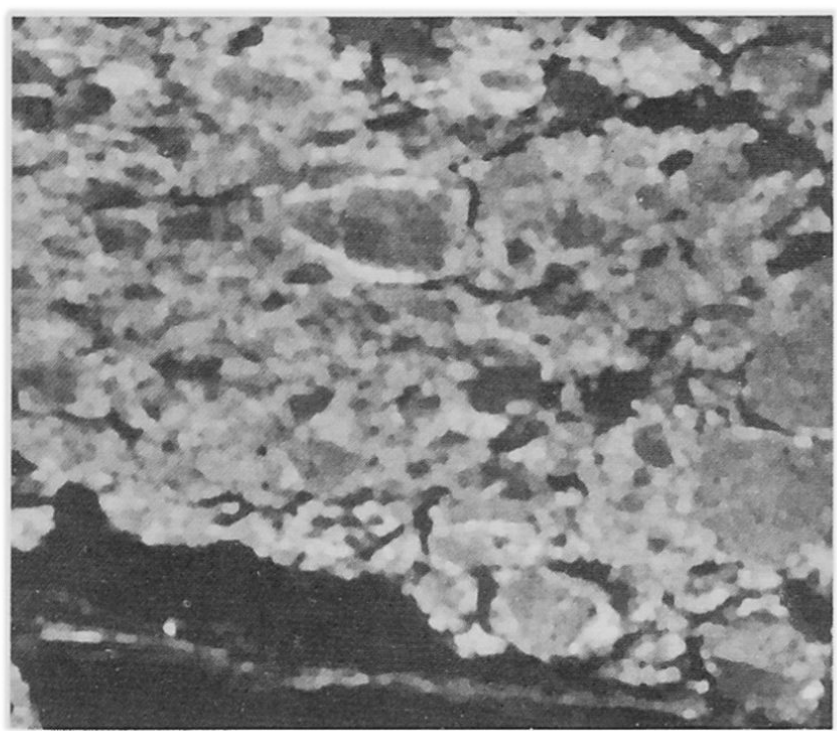

Abb. 3 Gefiltertes, vom Rauschen befreites Bild

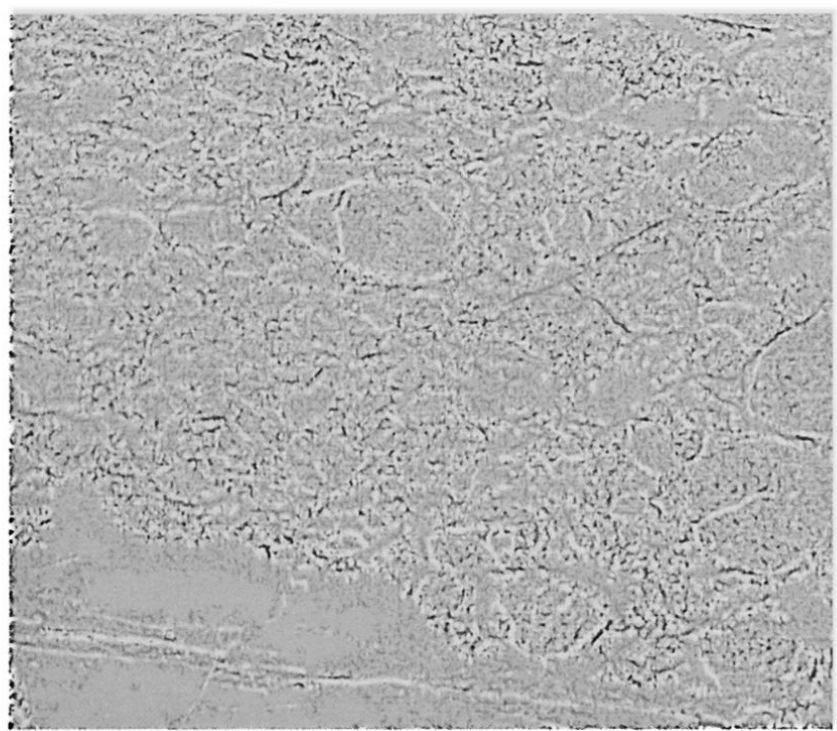

Abb. 4 Differenzbild, sog. "Texturbild"

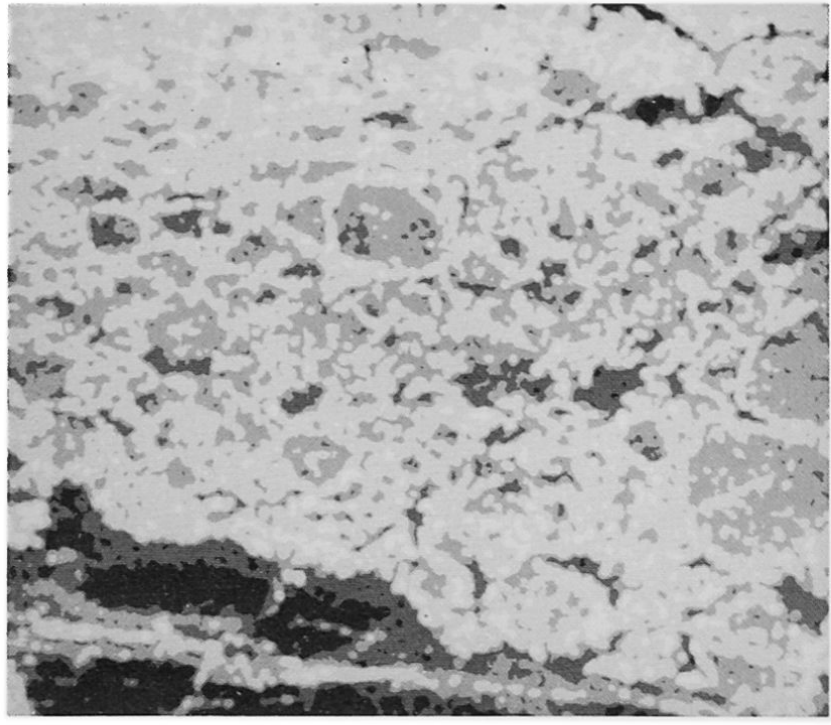

Abb. 5 Dargestellte Entropie des Texturbildes

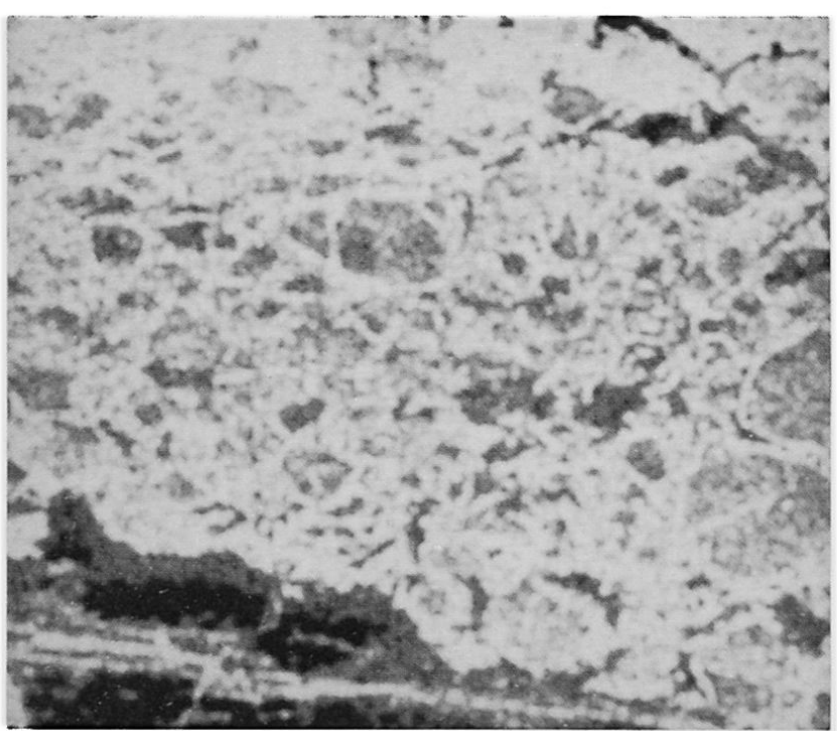

Abb. 6 Klassifiziertes Bild

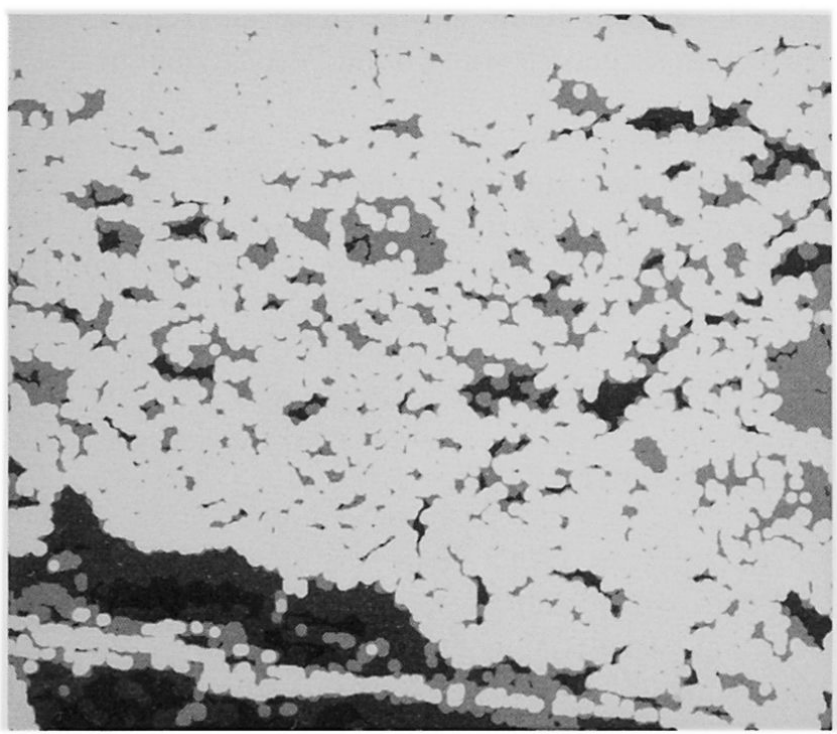

Abb. 7 Resultat der Schnellklassifikation 
Stellen stammen von Gebieten, die die Radarwellen gerichtet reflektieren, d.h. also Stellen mit offenem Wasser oder jüngerem Eis, das wie ein Spiegel wirkt.

Im nächsten Bild (Abb.3) sieht man die gefilterten Daten, d.h. die Eliminierung des lästigen "speckle»Effektes mit Hilfe eines speziellen isotropischen Filters, der die Werte innerhalb eines frei wählbaren Bildausschnittes mittelt.

Subtrahiert man die Originaldaten von diesem gefilterten Bild, so erhält man ein Differenzbild (Abb.4), das alle positiven und negativen Abweichungen vom ermittelten lokalen Mittelwert wiedergibt. Einigen wir uns nun darauf, daß alle diese Abweichungen auch als Textur bezeichnet werden können, so haben wir erstens den Grundstein zur digitalen Berechnung der Textur gelegt, die die flächenhafte Verteilung der Grauwerte mit ihren Regelmäßigkeiten und gegenseitigen Abhängigkeiten widerspiegelt, und zweitens eine Möglichkeit geschaffen, allfällige Information, die innerhalb der «speckle»-Erscheinung verborgen blieb, extrahieren zu können.

Berechnet man anschließend mit Hilfe der Abhängigkeitsstatistik (engl. co-occurrence) z. B. die Entropie dieses Texturbildes, so erhält man wie in Abb. 5 dargestellt folgendes Resultat: Die weißen Stellen bezeichnen Gebiete mit häufigen Wechseln, die dunklen Stellen Gebiete mit geringerer Dynamik, denn die Funktion der Entropie ist groß für gleiche relative Häufigkeiten und klein, wenn sie sehr verschieden sind. Damit stellt dieses Merkmal ein Maß dar für die Ordnung bzw. Unordnung innerhalb des Bildausschnittes, in dem die Abhängigkeitsmatrizen berechnet wurden. Obwohl dieses Bild noch große Ähnlichkeit mit dem ursprünglichen Bild besitzt, haben die Grauwerte dieses Bildes direkt überhaupt nichts mehr mit den ursprünglichen Grauwerten des Originalbildes gemeinsam, die die rückgestreuten Signale wiedergaben.

Dieses Entropiebild wurde anschließend in 5 empirisch festgelegte Klassen eingeteilt. Das Resultat ist in Abb. 6 festgehalten. Die 5 Klassen reichen von Schwarz (junges Eis und offene Wasserflächen) über 3 Graustufen (zweijähriges Eis, mehrjähriges Eis und mehrjähriges Eis mit deutlichen Kämmen) bis Weiß (saturiert, was auch mehrjährigem Eis entspricht). Dieses Resultat wurde mit Aufzeichnungen einer Expedition an Ort und Stelle verglichen und von den verantwortlichen Leitern als höchst ermutigend bezeichnet. Da diese Texturberechnungen auch bei optimaler Programmierung heute noch einen größeren Computer für Stunden in Anspruch nehmen, versucht man seit geraumer Zeit, die Rechenvorgänge zu optimieren und durch festverdrahtete sog. PipelineProzessoren ausführen zu lassen. Was vorher Stunden beanspruchte, kann so in Sekunden erledigt werden. Ein erstes Beispiel einer solchen Klassierung zeigt Abb.7, die nur noch 3 Klassen enthält. Dieses Verfahren ist in (HOLMES et al., 1984) ausführlicher beschrieben. Obwohl das Resultat noch einiges zu wünschen übrig läßt, kann doch eine hohe Übereinstimmung mit dem vorhergehenden Bild festgestellt werden. Für die Schiffscrew würde dies somit bedeuten, daß sie sich ihre Route im schwarzen Gebiet aussuchen muß. Auch wenn in diesem Artikel nur ein Anwendungsbeispiel vorgestellt wurde, muß der Vollständigkeit halber erwähnt werden, daß ähnliche Studien (NÜESCH, 1982) auch für landwirtschaftlich genutzte Flächen vorliegen.

\section{Schlußbemerkungen}

Im Rahmen dieses Artikels konnte es sich nur darum handeln, dem Leser einen kurzen Einblick in die Problematik der Verwendung von Radardaten zu vermitteln; für zusätzliche Information muß auf das Literaturverzeichnis hingewiesen werden.

Hinter dem Bildradar steckt eine hochentwickelte Technologie, die v. a. auf dem Boden der modernen Elektronik gewachsen ist.

Zwischen den Errungenschaften der Ingenieure, die dieses System konzipiert haben und stetig weiterentwickeln, und deren nutzbringender Anwendung klafft eine große Lücke. Wir stehen heute erst am Anfang einer intensiven Interpretationsphase.

Auf dem Weg zur operationellen Anwendung von Radarbildern sind noch sehr viele Hürden zu nehmen, wie z. B. ein besseres Verständnis der Wechselwirkung zwischen einfallender Strahlung und gemessenem Rückstreusignal sowie die Entwicklung von Algorithmen und Programmen, mit denen sich die riesigen Datenmengen erfolgreich bewältigen lassen. Ich bin aber zuversichtlich, daß die Forschung auf diesem Gebiet in naher Zukunft noch bessere Resultate liefern wird.

\section{Literaturverzeichnis}

COLWELL, R. N., ed. (1983): Manual of Remote Sensing, Volume I + II. American Society of Photogrammetry, Falls Church, Virginia, USA.

CONNERS, R.W., HARLOW, C.A. (1980): A Theoretical Comparison of Texture Algorithms, IEEE Transactions on Pattern Analysis and Machine Intelligence. Vol. PAMI-2, No. 3.

HARALICK, R.M., SHANMUGAM, K.S., DINSTEIN, I. (1973): Textural Features for Image Classification, IEEE Transactions on Systems. In: Man and Cybernetics, Vol. SMC-3, No. 6.

HOLMES, Q.A., NÜESCH, D.R., SHUCHMAN, R. A. (1984): Textural Analysis and Real-Time Classification of Sea-Ice Types Using Digital SAR Data. In: IEEE Transactions on Geoscience and Remote Sensing, Vol. GE-22, No. 2.

NÜESCH, D. R. (1982): Augmentation of Landsat MSS Data by Seasat SAR Imagery for Agricultural Inventories. In: Remote Sensing Series, Vol. 7, Department of Geography University of Zurich.

ULABY, F.T., MOORE, R.K., FUNG, A.K. (1981): Microwave Remote Sensing, Volume I + II. Addison-Wesley Publishing Company, Reading, Massachusetts, USA. 